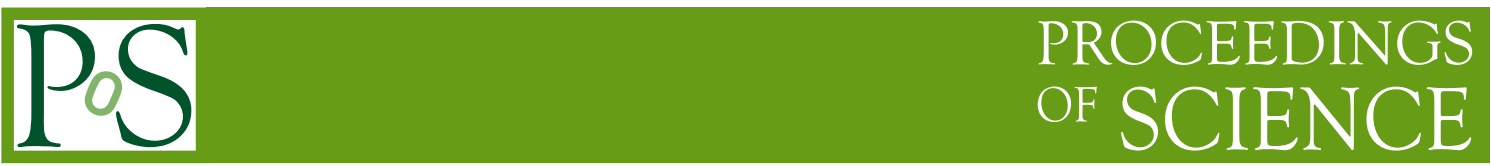

\title{
Ultimate spectrum of solar/stellar cosmic rays
}

\author{
Alexei Struminsky* \\ Space Research Institute, Profsoyuznaya st. 84/32, Moscow 117927, Russia \\ E-mail: astrumeiki.rssi.ru
}

We propose a physical approach to reconstruct the ultimate spectrum of solar/stellar cosmic rays (SCR) in a given point in the heliosphere (stellar sphere) basing on maximal value of magnetic field strength in active region and its characteristic linear dimension. An accelerator of given dimensions and magnetic field strength may accelerate to some finite energy for a given time (a maximal energy of SCR). We use the spectrum of SCR proposed by Syrovatsky (1961) for relativistic and non-relativistic energies normalizing it to galactic cosmic ray (GCR) intensity at the maximal SCR energy. Maximal values of SCR flux propagating in the heliosphere are determined by equilibrium between pressure of interplanetary magnetic field and dynamic pressure of SCR (Freier and Webber, 1963). We use the characteristic time of fluence accumulation obtained from the observations to derive the ultimate fluence of a single solar proton event (SPE). Our estimates are consisted with the observational upper limits on the strength of SPEs on the timescale of tens of millennia (Usoskin and Kovaltsov, 2012) and during the most active interval of $1945-1995$ during the last 2000 years (Cliver et al., 2014). The obtained numbers of $>30 \mathrm{MeV}$ and $>200$ $\mathrm{MeV}$ solar protons for a single proton event is not enough to explain the extreme particle event occurred in about $775 \mathrm{AD}$, a sequence of about twenty proton events may explain it. An extension of the model to cases of stellar flares is discussed.

The 34th International Cosmic Ray Conference,

30 July- 6 August, 2015

The Hague, The Netherlands

\footnotetext{
* Speaker.
} 


\section{Introduction}

Distributions of solar events by a value of one or another observational parameter are a base for prediction of their occurrence in the past and future. Recent observations of stellar mega-flares by the Kepler space craft showed that a scale of giant solar flares may exceed that reconstructed with the radiocarbon analysis (14C) in tree rings [四. These distributions should be limited by a maximal value of a chosen parameter due to physical reasons therefore their direct extrapolation to large values is impossible. Here we will discuss the ultimate (upper limit) spectrum of solar (stellar) protons (their intensities and fluencies), which might be observed in the heliosphere (stellar sphere), based on physical arguments. The upper limit spectrum was constructed recently in [Q]

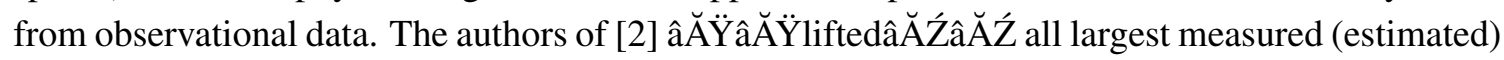
$\mathrm{I}(>\mathrm{Ep})$ values by approximately an order of magnitude in order to exceed the measuring errors and eliminate the methodical uncertainties.

Events with large proton fluence are generally associated with a sequence of activity as an active region crosses the central meridian of the sun generating a series of particle events and interplanetary disturbances [3]. A systematic survey of these very large omni-directional fluence solar proton events shows an association with two groups of solar source location. One group is west events, where the spacecraft is well connected to the solar active region by the interplanetary magnetic field, SCR propagate diffusively and their intensity time profiles have one maximum. Such events, their SCR fluxes and fluences we will call bellow diffusive. The other group is events located near the Earth-Sun line, they have the second maximum of SCR intensity, associated with propagating interplanetary magnetic field disturbances. In a case of strong disturbances the second maximum at energies below $100 \mathrm{MeV}$ is higher and has a large impact to the total fluence, the dominant mechanism of SCR propagation is the convection. Events from the second group, their SCR fluxes and fluences we will call below storm.

The probability of occurrence of solar particle event with large fluence varies approximately as the inverse square of the strength of the interplanetary magnetic field [团]. Authors [团] concluded that the observed dependence of the probability of occurrence of large - fluence SPEs on the strength of the IMF is unlikely to be due to propagation processes from Sun to Earth. The largest solar proton fluences are expected at low values of IMF strength, but the largest proton intensities are expected under disturbed conditions of IMF (its highest values). The frequency distribution of the most common solar proton events does not represent the extreme events. Solar proton events with $>30 \mathrm{MeV}$ omni - directional fluence exceeding $6 \cdot 10^{9} \mathrm{~cm}^{-2}$ are very rare [四]. Therefore the extreme events should be close to their physical limits.

\section{The source spectrum and propagation limits}

Let us assume that solar protons registered by neutron monitors are accelerated in a flare site. An important parameter in the study of particle acceleration mechanisms is the ratio of the protons to electrons. Observations of solar electrons and protons in the interplanetary medium discussed in [5] suggests that for $0.5-1.1 \mathrm{MeV}$ electrons and $10 \mathrm{MeV}$ protons are accelerated by the same mechanism. Shih et al. [6] considered all RHESSI measurements from 2002 to 2009 (29 flare events) of the $2.223 \mathrm{MeV}$ neutron capture line and $>0.3 \mathrm{MeV}$ bremstrahlung continuum emissions 
and concluded that none of the current acceleration theories that treat both ion and electron acceleration predict, a priori, such a closely constrained ratio of $>0.3 \mathrm{MeV}$ electrons to $>30 \mathrm{MeV}$ protons when integrated over a flare, nor do they predict a close correlation of $>50 \mathrm{keV}$ electron acceleration when the $>30 \mathrm{MeV}$ proton acceleration exceeds a threshold. The acceleration mechanism is still unknown at present, therefore for our estimates we choose a spectrum of solar cosmic rays proposed by Syrovatsky [四].

This spectrum was derived from general principles of thermodynamics, unrelated to specific parameters of acceleration mechanism. Solar cosmic rays are considered as a gas with a constant ratio of heat capacities (which is 5/3 for non-relativistic gas and $4 / 3$ for relativistic gas) adiabatically compressed by constant external magnetic field. Both electrons and protons should be accelerated effectively, their differential spectrum is a power law with index 3.5 for non-relativistic and 5 for ultra - relativistic energies. A transition from non - relativistic to ultra - relativistic energies should be smoothed.

Solar cosmic rays escape from the acceleration region into the heliosphere if their pressure exceeds a pressure of the magnetic field. From this pressure balance we may estimate a maximal density of accelerated particles $n$, their total number $N$ in an acceleration region with characteristic dimensions $L$ and a total fluence $F$ at a distance $R$. We will get $n=\frac{B^{2}}{8 \pi E}=8.3 \cdot 10^{6} \mathrm{~cm}^{-3}$ and $N=n L^{3}=8.3 \cdot 10^{36}$ protons for a proton energy $E=30 \mathrm{MeV}$, an accelerator dimension $L=10^{10}$ $\mathrm{cm}$, a magnetic field strength $B=100 \mathrm{Gs}$, which implies $F=\frac{N}{4 \pi R^{2}}=2.9 \cdot 10^{9} \mathrm{~cm}^{-2}$ at a distance $R$ $=1.5 \cdot 10^{13} \mathrm{~cm}$. Since SCR propagate within a finite spatial angle the ultimate fluence can be about one order higher and the obtained number corresponds to SCR observations near the Earth.

Maximal values of solar proton intensity observed at different energies in the interplanetary space roughly correspond to the source spectrum. To get the ultimate spectrum near the Earth we normalize the source spectrum to the spectrum of galactic cosmic rays at the maximal possible energy of solar protons. Otherwise we do not have a possibility to know are solar protons accelerated or not up to ultra-relativistic energies. The maximal energy of proton acceleration we may estimate basing on characteristic parameters of the acceleration region. For a linear dimension $L=10^{10} \mathrm{~cm}$, a magnetic field strength $B=100 \mathrm{Gs}$ and a plasma velocity $V=100 \mathrm{~km} / \mathrm{s}$ we will get $E=\frac{1}{c} e V L=100 \mathrm{GeV}$. Svestka [ [ $]$ ] calculated a maximal energy of accelerated electrons about several tens of $\mathrm{MeV}$ accounting energy losses. It is adopted that the high energy cut of solar electrons is $10 \mathrm{MeV}$. Therefore, for protons it should be about $20 \mathrm{GeV}$ that roughly corresponds to observations.

The blue lines in fig. W show the high energy part of the ultimate solar spectrum. Left and right blue lines are different by their normalization, the left spectrum is normalized to the upper limit flux of solar protons at $10 \mathrm{MeV}$ [D] and the left is normalized to the GCR spectrum at $20 \mathrm{GeV}$. The intensity of SCR may not be much higher at $20 \mathrm{GeV}$, otherwise the maximal energy of solar protons should be larger. A transition from relativistic to non-relativistic spectra was assumed at $2 \mathrm{GeV}$ in both cases. The lower energy part of the spectrum would be determined by propagation limits. The spectrum proposed in [0] (red line in fig. 四) looks unreasonably soft and hard at energies below and higher $200 \mathrm{MeV}$ respectively, there is an intersection of the red and right spectra at about 2 $\mathrm{GeV}$. To our opinion the spectrum [[] does not represent the ultimate spectrum of solar protons at the Earth orbit.

The same physics - a magnetic field structure may keep particles inside the structure if the 


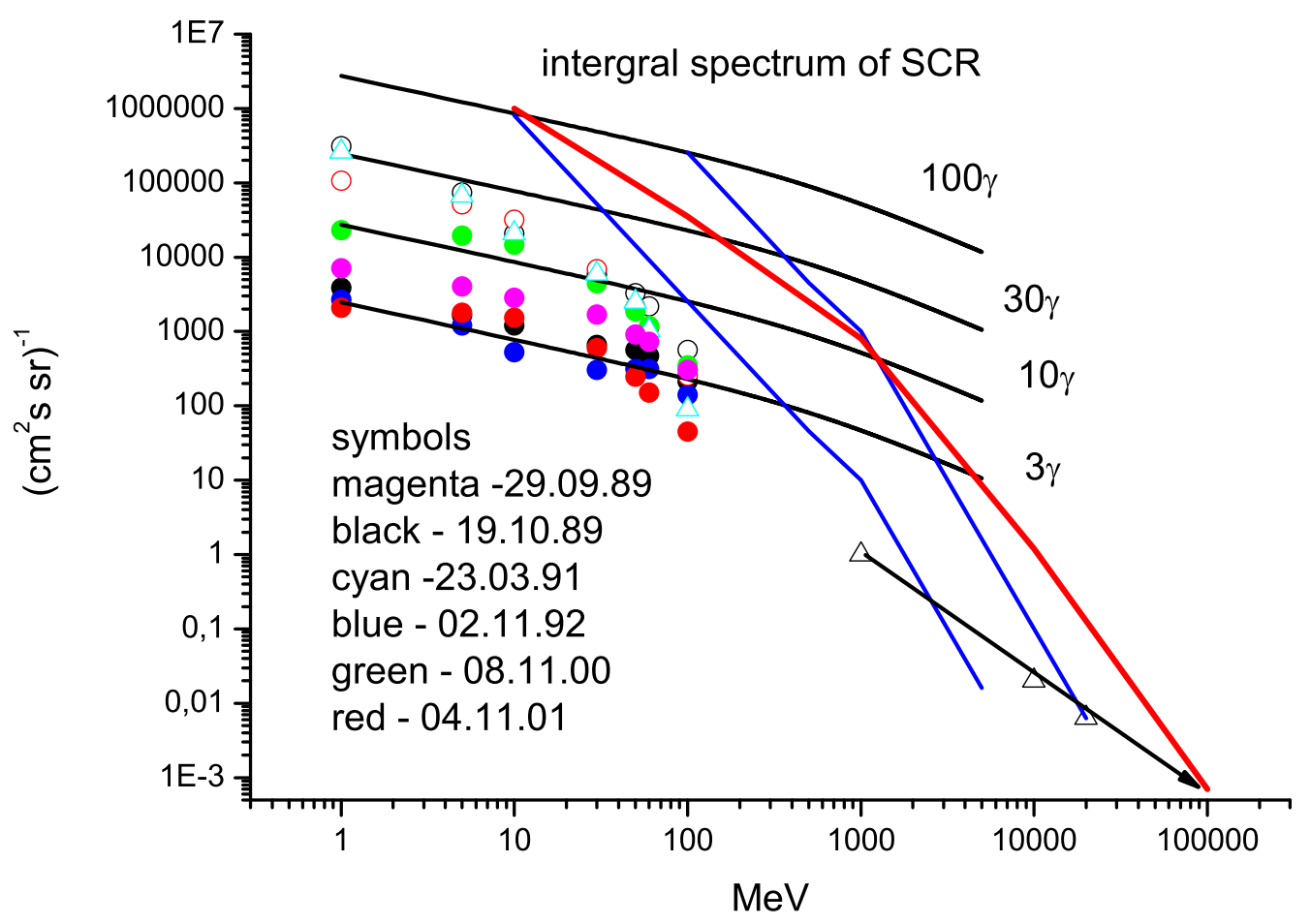

Figure 1: Closed and open symbols - maximal diffusive and storm proton intensities for different events. Black curves - propagation limits [可] for different values of the IMF strength. Blue lines - spectra of non relativistic and relativistic solar protons [四] with different normalization. Red - the upper limit spectrum of solar protons proposed in [D]. Black line with open triangles - the spectrum of GCR.

magnetic field pressure is greater than a particle pressure - determines propagation limits as proposed in [Q]. From this pressure balance we get a maximal flux of solar protons $J$ with energy $E$ and velocity $V$ in a given point in the heliosphere with magnetic field strength $B$

$$
J=\frac{B^{2} V}{32 \pi^{2} E}
$$

The black curves in fig. $\mathbb{W}$ show these propagation limits for different values of the IMF strength, which are lower and upper limits of the IMF strength at the Earth orbit. We do not know exactly a radial dependence of the IMF strength, a relation between characteristic values of magnetic field in active region and solar wind. Maximal values of solar proton flux registered by GOES proton detectors during different events (symbols in fig. 四) roughly corresponds to these estimates, diffusive and storm particles are observed at lower and higher values of IMF strength respectively. 


\section{Discussion: ultimate fluxes and fluences}

In the previous section we have got estimates of ultimate solar proton fluxes. In order to get ultimate fluences we will at first analyze experimental data presented in fig. \. Note that fluences may be accumulated in two steps, which correspond to diffusive and storm particles, within a finite time. Table $\mathbb{W}$ presents maximal values of $>30 \mathrm{MeV}$ proton fluxes and fluences. A ratio of fluence to maximal flux give us a characteristic time, this time is similar for different events. The diffusive fluences were accumulated during about $1000 \mathrm{~min}$ ( 16.7 hours), but about $250 \mathrm{~min}$ ( 4.2 hours) for storm fluences. These characteristic times are not very different for proton energies within 1-100 $\mathrm{MeV}$. We will use the diffusive characteristic time $1000 \mathrm{~min}$ to get the ultimate fluences, so this will be an upper estimate.

Table $\square$ shows ultimate fluxes $J$ and ultimate omni - directional fluences $F$ calculated for $>30$ $\mathrm{MeV}$ and $>200 \mathrm{MeV}$ solar protons and different values of IMF strength. The fluences of $>30 \mathrm{MeV}$ protons obtained from the ultimate spectrum and characteristic time corresponds to that estimated from the release condition supporting our choice of the spectrum. We add the results for $>200 \mathrm{MeV}$ protons, because the integral fluence of an SPE above $200 \mathrm{MeV}$ is related to the production of the cosmogenic isotopes $14 \mathrm{C}$ and $10 \mathrm{Be}$ in the Earth atmosphere, independently of the assumptions on the energy spectrum of the event
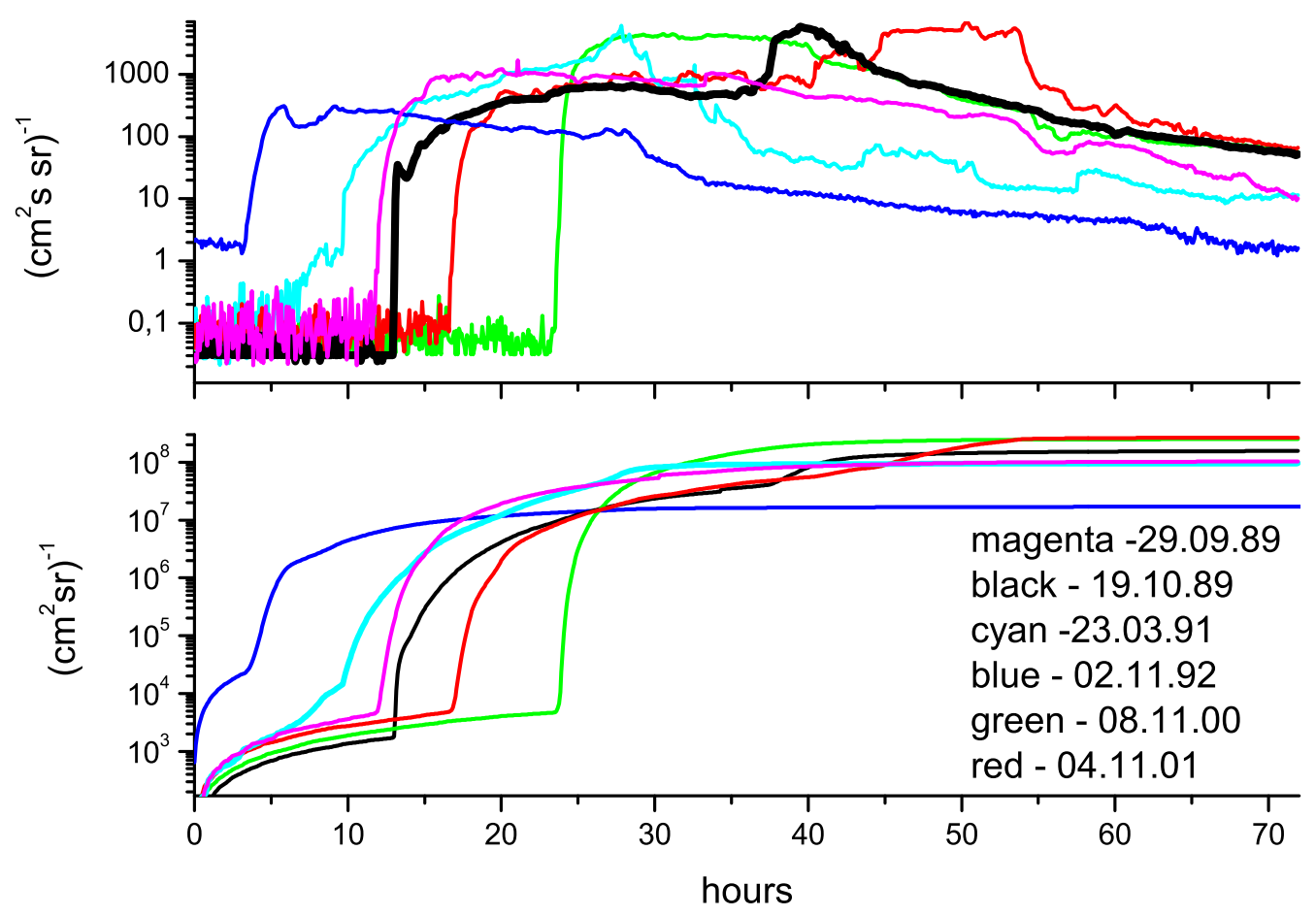

Figure 2: Time profiles of solar proton $>30 \mathrm{MeV}$ intensities and accumulated fluencies in the considered events. 


\begin{tabular}{ccccccc}
\hline & Sep 29, & Oct 19, & Mar 22, & Nov 02, & Nov 08, & Nov 04, \\
& 1989 & 1989 & 1991 & 1992 & 2000 & 2001 \\
\hline FD $(>30 \mathrm{MeV})$ & $1.0 \mathrm{E} 8$ & $4.0 \mathrm{E} 7$ & - & $1.7 \mathrm{E} 7$ & $2.5 \mathrm{E} 8$ & $5.5 \mathrm{E} 7$ \\
$\mathrm{JD}(>30 \mathrm{MeV})$ & 1690 & 665 & - & 307 & 4440 & 980 \\
\hline $\mathrm{TD}=\mathrm{FD} / \mathrm{JD}$ & 1005 & 988 & - & 935 & 945 & 938 \\
\hline $\mathrm{FS}(>30 \mathrm{MeV})$ & - & $9.4 \mathrm{E} 7$ & $9.6 \mathrm{E} 7$ & - & - & $2.7 \mathrm{E} 8$ \\
$\mathrm{JS}(>30 \mathrm{MeV})$ & - & 5826 & 6010 & - & - & 6870 \\
\hline $\mathrm{TS}=\mathrm{FS} / \mathrm{JS}$ & - & 267 & 264 & - & - & 647 \\
\hline
\end{tabular}

Table 1: Maximal values of solar proton fluxes $\mathrm{J}\left(\mathrm{cm}^{-2} \mathrm{~s}^{-1} \mathrm{sr}^{-1}\right)$ and fluences $\mathrm{F}\left(\mathrm{cm}^{-2} \mathrm{sr}^{-1}\right)$ observed by GOES in the considered events for diffusive (D) and storm (S) maxima of proton intensity. A characteristic time $\mathrm{T}(\mathrm{min})$ of fluence accumulation for diffusive and storm protons.

\begin{tabular}{ccccc}
\hline & $3 \mathrm{nT}$ & $10 \mathrm{nT}$ & $30 \mathrm{nT}$ & $100 \mathrm{nT}$ \\
\hline $\mathrm{J}(>30 \mathrm{MeV})$ & $4.4 \mathrm{E} 2$ & $4.9 \mathrm{E} 3$ & $4.4 \mathrm{E} 4$ & $4.9 \mathrm{E} 5$ \\
$\mathrm{~F}(>30 \mathrm{MeV})$ & $2.8 \mathrm{E} 6$ & $3.1 \mathrm{E} 7$ & $2.8 \mathrm{E} 8$ & $3.1 \mathrm{E} 9$ \\
\hline $\mathrm{J}(>200 \mathrm{MeV})$ & $1.5 \mathrm{E} 2$ & $1.7 \mathrm{E} 3$ & $1.5 \mathrm{E} 4$ & $1.7 \mathrm{E} 5$ \\
$\mathrm{~F}(>200 \mathrm{MeV})$ & $9.4 \mathrm{E} 5$ & $1.1 \mathrm{E} 7$ & $9.4 \mathrm{E} 7$ & $1.1 \mathrm{E} 9$ \\
\hline
\end{tabular}

Table 2: Ultimate fluxes $\mathrm{J}\left(\mathrm{cm}^{-2} \mathrm{~s}^{-1} \mathrm{sr}^{-1}\right)$ and ultimate omni - directional fluences $\mathrm{F}\left(\mathrm{cm}^{-2}\right)$ estimated for different values of IMF strength.

According to Usoskin and Kovaltsov [ய0] 19 SPE candidates with $F(>30 \mathrm{MeV})=1-1.5 \cdot 10^{10}$ $\mathrm{cm}^{-2}$ and no events with $F(>30 \mathrm{MeV})>2 \cdot 10^{10} \mathrm{~cm}^{-2}$ (50 times the SPE of 1956 February 23 ) are identified since $1400 \mathrm{AD}$ in the annually resolved 10Be data. A strong SPE related to the Carrington flare of $1859 \mathrm{AD}$ is not supported by the data. These values serve as observational upper limits on the strength of SPEs on the timescale of tens of millennia, they are consistent with our estimates of physical limits presented in Table $\square$ if we assume that the fluence resulted in the production of the cosmogenic isotopes was accumulated during several single events occurred within three months. The considerations of Cliver et al. [U]] challenge a suggestion that the 775 event is likely of solar origin. This event could originate from high-energy irradiation of the atmosphere by a Galactic gamma-ray burst [133]. A solar source for the AD 775 event would require the proton fluence $F(>30 \mathrm{MeV})=8 \cdot 10^{10} \mathrm{~cm}^{-2}, 10$ times larger than that of the strongest 3 month interval of SPE activity in the modern era.

In [[D] it was evaluated that extreme SPEs with $F(>200 \mathrm{MeV})>10^{10} \mathrm{~cm}^{-2}$ occur no more frequently than once per 10000 - 15000 years. This fluence is about one order higher than our upper estimate. Such events may occur only if the solar parameters are different, i.e. much earlier, when the Sun was rotated much more quickly and solar magnetic field might be expected higher than its current value. At present the probability of such events is zero.

If a stellar magnetic field is a result of the dynamo mechanism, then the stellar rotation is a source of magnetic field energy. Due to stellar magnetic activity stars loss their rotation energy. Young stars rotating more quickly should have greater values of magnetic field, flare frequency 
and energy. Increasing of magnetic field in active region by about one order will lead to one order increasing of maximal energy of particle acceleration and two orders increasing of ultimate fluxes of SCR. The ultimate spectrum of SCR for the maximal energy of proton acceleration 200 $\mathrm{GeV}$ and different values of the IMF strength is presented in fig. [3. Stellar cosmic rays are considered as an additional GCR source, they might produce the main source of positrons observed by PAMELA [ [14]]. In future we suppose to use data available on stellar flares and estimate their possible contribution to GCR at different energies.

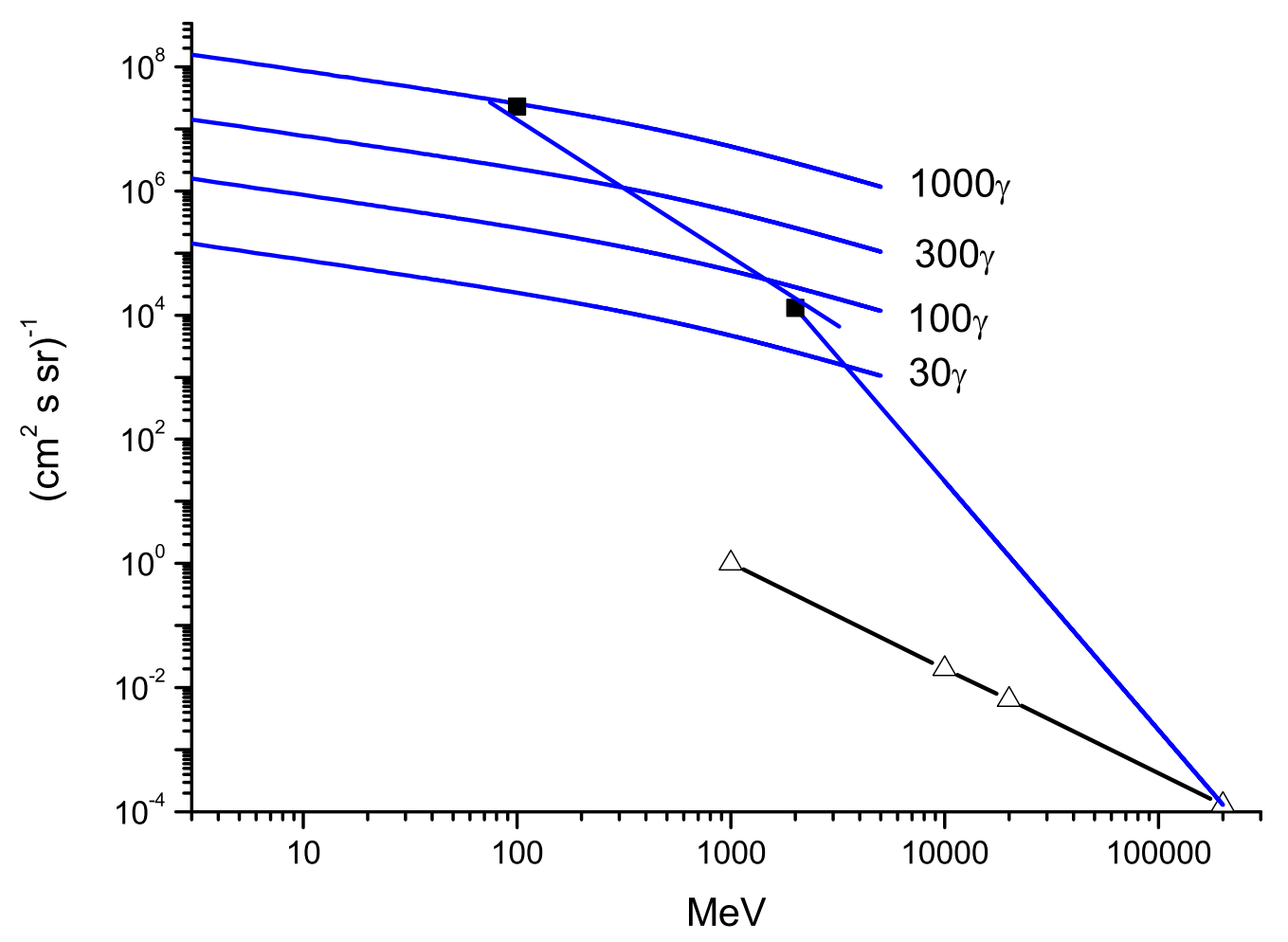

Figure 3: Blue lines - the ultimate spectrum of SCR for stellar flares. Values of magnetic field strength are enhanced by one order in comparison with solar flares (fig/ W). Black line with open triangles - the spectrum of GCR.

\section{Conclusions}

1. The ultimate spectrum of SCR near the earth orbit was reconstructed basing on the source spectrum [ $[\mathbf{\square}]$ and propagation limits in the interplanetary space [ [] . The spectrum has two knees, one is at about $200 \mathrm{MeV}$ due to propagation conditions and another at about $2000 \mathrm{MeV}$ corresponds to transition from relativistic to non-relativistic regimes of particle acceleration.

2. The spectrum proposed in [] (red line in fig. 四) looks unreasonably soft and hard at energies lower and higher $200 \mathrm{MeV}$ respectively, it does not account transition to relativistic energies 
and energy losses. Therefore to our opinion it does not represent the ultimate spectrum of solar protons at the earth orbit. There is two points of intersection with our ultimate spectrum at about $10 \mathrm{MeV}$ and $2 \mathrm{GeV}$.

3. We use the characteristic time of fluence accumulation obtained from the observations to derive an ultimate fluence of a single solar proton event (SPE). Our estimates are consisted with the observational upper limits on the strength of SPEs on the timescale of tens of millennia [एU] and during the most active interval 1945 - 1995 of the last 2000 years [ए]].

4. The obtained numbers of $>30 \mathrm{MeV}$ and $>200 \mathrm{MeV}$ solar protons for a single proton event is not enough to explain the extreme particle event occurred in about $775 \mathrm{AD}$ basing on the tree-ring chronology, a sequence of about twenty proton events may explain it.

\section{References}

[1] H. Hudson Solar extreme events, eprint (2015) arXiv: 1504.04755.

[2] L. I. Miroshnichenko, E. V. Vashenyuk, and J. A. Perez-Peraza, Solar cosmic rays: 70 years of ground - based observations, Geomagnetism and Aeronomy, 53 (2013) 541.

[3] M.A. Shea, D.F. Smart, Solar proton fluxes as a function of the observation location with respect to the parent solar activity, Advances in Space Research, 17 (1996) 225.

[4] D.F. Smart, M.A. Shea, H.E. Spence, L. Kepko, Two groups of extremely large $>30$ MeV solar proton fluence events, Advances in Space Research, 37 (2006) 1734.

[5] R. Ramaty, C. Paizis, S.A. Colgate, G.A. Dulk, P. Hoyng, J.W. Knight, R.P. Lin, D.B. Melrose, F. Orrall, P.R. Shapiro, Energetic particles in solar flares, Solar flares: A monograph from Skylab Solar Workshop II. Colorado Associated University Press, Boulder 1980.

[6] A. Y. Shih, R. P. Lin, D.M. Smith, RHESSI Observations of the Proportional Acceleration of Relativistic $>0.3 \mathrm{MeV}$ Electrons and $>30 \mathrm{MeV}$ Protons in Solar Flares, The Astrophysical Journal, 698 (2009) L152.

[7] S.I. Syrovatsky, On spectrum of galactic and solar cosmic rays, ZhETPh, 40 (1961) 1788 (in Russian).

[8] Z. Svestka, The phase of particle acceleration in flare development, Solar Physics, 13 (1970) 471.

[9] P.S. Freier, W.B. Webber, Exponential rigidity spectrums for solar-flare cosmic rays, Journal of Geophysical Research, 68 (1963) 1605.

[10] I.G. Usoskin, G. A. Kovaltsov, Occurrence of extreme solar particle events: assessment from historical proxy data, The Astrophysical Journal, 757 (2012) 92.

[11] E. W. Cliver, A. J. Tylka, W. F. Dietrich, A. G. Ling, On a solar origin for the cosmogenic nuclide event of 775 A.D. The Astrophysical Journal, 781 (2014) 32.

[12] G.A. Kovaltsov, I.G. Usoskin, E.W. Cliver, W.F. Dietrich, A.J. Tylka, Fluence Ordering of Solar Energetic Proton Events Using Cosmogenic Radionuclide Data, Solar Physics, 289 (2014) 4691.

[13] A. K. Pavlov, A. V. Blinov, A. N.Konstantinov, V. M. Ostryakov, G. I. Vasilyev, M. A. Vdovina, P. A. Volkov AD 775 pulse of cosmogenic radionuclides production as imprint of a Galactic gamma-ray burst Monthly Notices of the Royal Astronomical Society, 435 (2013) 2878.

[14] Yu.I. Stozhkov, The anomalous PAMELA effect and its explanation, Bulletin of the Russian Academy of Sciences. Physics, 75 (2011) 323. 